\title{
MODELLING THE CO-EVOLUTION OF TRADE AND CULTURE IN PAST SOCIETIES
}

\author{
Simon Carrignon \\ Jean-Marc Montanier \\ Xavier Rubio-Campillo \\ Barcelona Supercomputing Center \\ Carrer de Jordi Girona, 29, \\ Barcelona, 08034, SPAIN
}

\begin{abstract}
This paper presents a new framework to study the co-evolution of cultural change and trade. The design aims for a trade-off between the flexibility necessary for the implementation of multiple models and the structure necessary for the comparison between the models implemented. To create this framework we propose an Agent-Based Model relying on agents producing, exchanging and associating values to a list of goods. We present the key concepts of the framework and two examples of its implementation which allow us to show the flexibility of our framework. Moreover, we compare the results obtained by the two models, thus validating the structure of the framework. Finally, we validate the implementation of a trading model by studying the price structure it produces.
\end{abstract}

\section{INTRODUCTION}

Cultural change comprises the collection of processes that promotes or inhibits the spread of information by social interaction within a population (Boyd and Richerson 2005). An increasing number of social scientists are using an evolutionary framework to model cultural change (Henrich and McElreath 2003). This approach aims at fostering the development of transdisciplinary efforts designed to understand cultural change.

Within the studies done in the evolutionary framework, a cultural phenomenon (such as music) is viewed as a collection of traits (such as musical genres). Multiple biases (mechanism favouring the use of a cultural trait over an other) can explain the fact that certain of the cultural traits are transmitted more readily than the others. Among the biases studied, some can be explained by the intrinsic properties of a trait (how beneficial it is for the individual using it), while others are explained by the frequency of these traits (how popular a trait is in the culture).

Multiple models can be proposed to represent cultural changes, one of them being the neutral model (Neiman 1995). Within this type of model it is assumed that a trait does not bias the fitness of the individual that acquires it. It therefore means that no bias modifies the rate of transmission of the cultural traits, and that their success will depend only on their frequency in the population. Within analysis of real data, a neutral model produces a distinctive type of frequency distribution of cultural traits termed power law.

The power law can be replicated within a virtual setup thanks to a simple random copying transmission mechanism (Bentley, Hahn, and Shennan 2004): an individual will copy the traits of a randomly chosen individual with a given probability. This copy can potentially introduce some errors in the acquired trait, which account for innovation processes. The individual will in turn continue to spread these cultural traits which will be further adopted by other individuals. This basic model can be enriched by several additional processes both in the innovation (Schillinger et al. 2014, Solé et al. 2013, Ziman 2003) and the transmission (Heyes 1994, Henrich and McElreath 2003). Unbiased transmission works as a baseline for 
identifying frequency-dependent biases: if evidence has higher tendency to copy the most common trait it is known as conformism, while the opposite is defined as anti-conformism.

The archaeological record allows the researchers to identify frequency-dependence biases on cultural transmission over long-term trajectories (Lipo and Madsen 2001, Shennan and Wilkinson 2001, Steele, Glatz, and Kandler 2010). However, the fact that material culture recovered from archaeological contexts is noisy and fragmented presents some challenges on the validity of the method (Kandler and Shennan 2013, Porčić 2014, Crema, Edinborough, Kerig, and Shennan 2014).

This work explores the impact of a crucial element on the transmission of material culture: trade. Networks of good exchanges are being increasingly recognised as key elements that structured ancient societies (Temin 2001, Remesal et al. 2014, Brughmans 2010). The scenarios where this process emerges suggest a complex bias in the selection of cultural traits, which at the same time are also identified as economic goods (Bentley, Lake, and Shennan 2005, Macmillan and Huang 2008). Trasmission is not neutral anymore, as different prices for each good will introduce a dynamic content bias. This affects the frequency of the good within the population, which in turn modifies its price following a co-evolutionary dynamic.

These dynamics are studied using an Agent-Based Model (ABM), a type of simulation particularly useful for studying non-linear dynamics in heterogeneous environments within an evolutionary perspective (Lake 2014). More precisely we propose a framework that can be implemented in multiple ways depending on the model tested. Next section defines the framework, which is based on the basic processes found in evolutionary models of cultural change. Next, we define the implementations used to explore the dynamics of the created framework. In the following section we analyse the results obtained with these two implementations. Finally, the concluding remarks discuss further possibilities of the presented framework.

\section{FRAMEWORK DESCRIPTION}

To explore the co-evolution between trade and cultural change we have developed a framework where the different agents produce and trade goods to which they assign variable values. The model is composed of a population Pop of $m$ agents, each defined by 2 vectors of size $n$. The first corresponds to the quantity of each good owned by the agent $i$ :

$$
\forall i \in \text { Pop }, \quad Q^{i}=\left(q_{1}^{i}, \cdots, q_{n}^{i}\right)
$$

where $Q^{i}$ is the total list of possessions of agent $i$, and $q_{j}^{i}$ is the number of goods of type $j$ that agent $i$ possesses.

The second vector reflects the estimation of the value of a good made by an agent $i$ :

$$
\forall i \in \text { Pop }, \quad V^{i}=\left(v_{1}^{i}, \cdots, v_{n}^{i}\right)
$$

where $V^{i}$ is the total list of estimated values of agent $i$, and $v_{j}^{i}$ is the value that agent $i$ associates to one unit of a good of type $j$.

On top of these elements five processes are used: production, consumption, cultural transmission, innovation and trade. The production process describes the creation of goods by the agent. Once a good is produced by an agent $i$ it is added to its quantity vector $\left(Q^{i}\right)$. The consumption strategy of these goods is defined in the consumption process which decreases the number of goods in the vector $\left(Q^{i}\right)$. In this article, all goods are completely consumed at each iteration for all the models tested. The trade process models the exchange of goods between the agents which results in a modification of the quantity vectors $\left(Q^{i}\right)$. The amount of goods exchanged is computed by the agents involved in the trade, within the trade process, based on their value vectors $\left(V^{i}\right)$. Within the cultural transmission process an agent $i$ can copy the entire value vector $\left(V^{j}\right)$ of an agent $j$, where $j \neq i$. Finally, the innovation process also modifies the value vector $V^{i}$ of an agent, but it differs from the cultural transmission process in that the modification is done without reference to the other agents.

The scheduling of the five processes is described in algorithm 1 along with the vectors modified by each of these processes. On lines 3 and 4 all agents of the population are initialised with empty quantity 
vectors and random values. The code used to update the status of each agent at each iteration is presented between the lines 8 and 22. One can note that each of the five processes is executed synchronously by all agents. Moreover, the trade process is called at each iteration while the cultural transmission and innovation processes are executed only every CulturalStep. The idea behind this is to perform the cultural transmission based on a score that reflects the performance of the agent and not only one lucky or unlucky trading round. The timestep number used in the figures of this article refers to the number of times the cultural transmission and innovation processes are called.

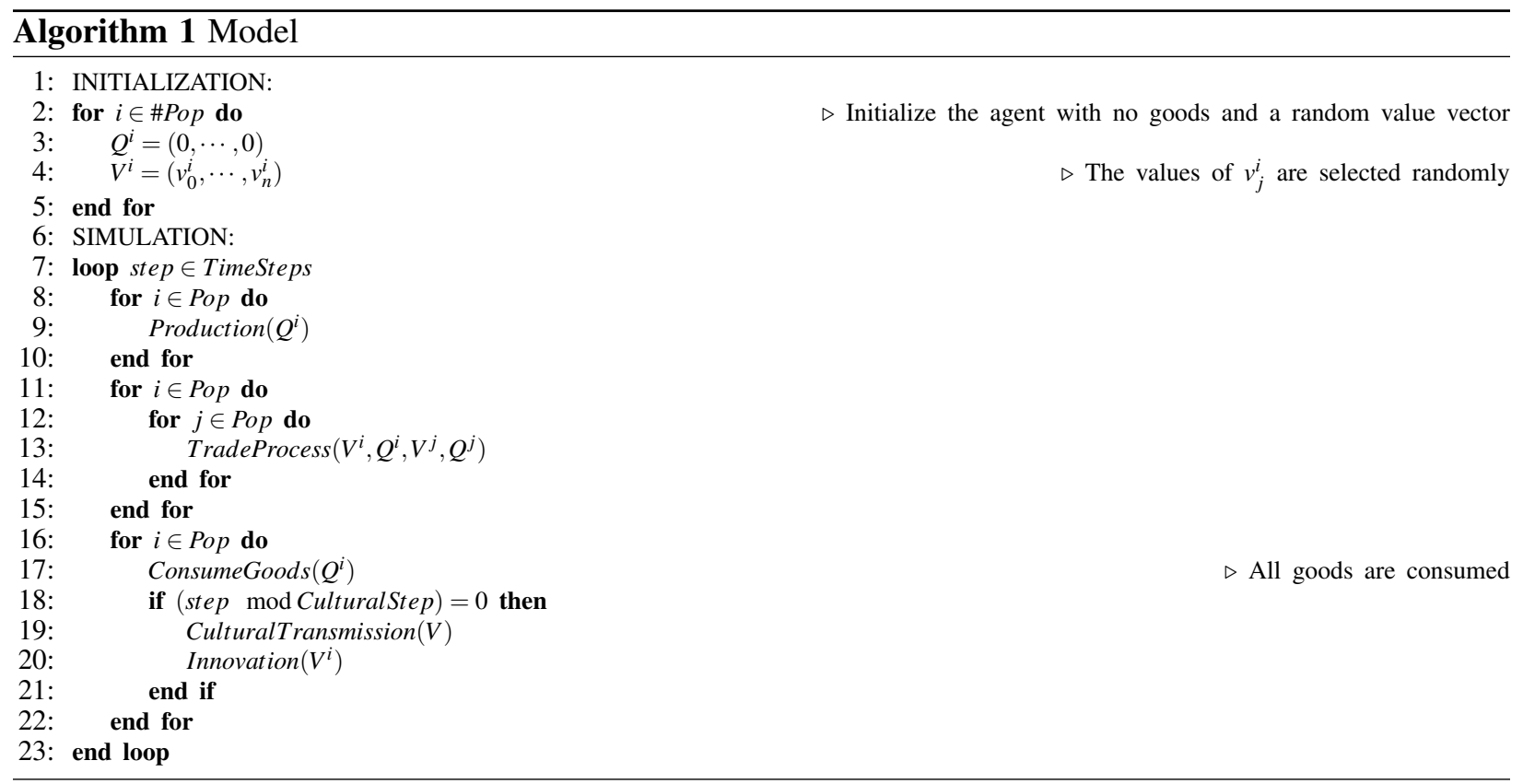

In order to validate our model we first reproduce common results from the literature on cultural transmission. We then show that it is possible to transform our model to fit processes that are economically sound, i.e. the model should show the convergence optimal values such as shown in (Gintis 2006). To achieve these two goals, we have designed for each one a specific set of implementations of the five core processes (production, consumption, trade, cultural transmission and innovation).

\subsection{Neutral Configuration}

The first scenario is designed to reproduce unbiased transmission, where each good is a cultural trait without intrinsic positive or negative weight (Bentley, Hahn, and Shennan 2004, Bentley, Lake, and Shennan 2005, Mesoudi and Lycett 2009). Under this hypothesis, the processes of production and trade are not relevant, and as a consequence, they do not modify the content of the quantity vectors of the agents.

Unbiased cultural transmission is implemented using "random copy": each agent has a low probability to pick randomly one agent among all and copy its vector of values. The innovation process, termed "unbounded", is triggered with a low probability $(\mu)$ and draw a new random value to replace an element $v_{j}^{i}$.

The neutral hypothesis states that the "random copy" transmission and the "unbounded" innovation process used under a fixed population size leads to a distribution of frequency of cultural variants termed power law. This distribution is characterized by a small number of very frequent traits and a large number of rare traits. The main difference with similar distributions, such as exponential distribution, is that the rare traits are far from being absent of the distribution, i.e. the tail of the distribution is large. This distribution is formalised as :

$$
P(v)=C / v^{\alpha}
$$


where $v$ is the number of times a variant has been repeated, $P(v)$ the probability to find that variant, $C$ a constant, $\alpha$ a variable describing the slope of the curve obtained. We will therefore attempt to fit as well as possible the results obtained with this set of implementations to the "power law" distribution by modifying the $\alpha$ parameter.

\subsection{Trading Biased Configuration}

In the second scenario, we are interested in the exchange of goods between agents in a barter process where each agent can choose its prices of exchange. We want to implement simple processes leading to the convergence of all prices to values acceptable by all agents, i.e. we would like to observe, at the end of an experiment, all the agents using a set of prices which allow them to trade efficiently.

Production Each agent produces one good. The type of good produced by an agent $i$ is assigned to it at the beginning of the simulation, does not change through the simulation, and is referred to as produced $^{i}$. At each time step, each agent, produces a number of units (of its production good) equal to the number of goods, which ensures that enough is owned to be traded for other goods. Moreover, when an agent consumes its own production good, it does not impact its inventory.

Cultural transmission Social learning is here biased towards the agents which are the best at trading, and is therefore termed "success bias". To achieve this bias, the cultural transmission mechanism used takes into account the value vector of the other agents and relies on two new notions: need and score.

The need is a quantity of good that each agent tries to obtain. This quantity is different for each good but the need for a good is the same for all agents:

$$
N=\left(n_{1}, \cdots, n_{r}\right)
$$

The score $s_{j}^{i}$ of an agent $i$ reflects the ability of this agent to obtain the quantity of good $j$ it needs. It is maximum when the quantity $q_{j}^{i}$ that an agent $i$ owns of the good $j$ is equal to the need $n_{j}$ for the good $j$ and lowers proportionally to the distance between the need vector and the quantity vector. It is formally computed as follows for agent $i$ and the good $j$ :

$$
s_{j}^{i}= \begin{cases}s_{\max }=1 & \text { if } q_{j}^{i}=n_{j} \\ 1-\frac{\left|q_{j}^{i}-n_{j}\right|}{\sqrt{\left|\left(q_{j}^{i}\right)^{2}-\left(n_{j}\right)^{2}\right|}} & \text { if } q_{j}^{i} \neq n_{j}\end{cases}
$$

This function ensures that each good has the same weight in the final score, i.e. managing to get only the right amount of a good with a high "need" value will not give a better score to the agent. The complete score of an agent $i$ is termed $s^{i}$ and corresponds to the sum of the $s_{j}^{i}$. An agent will choose from whom the price vector should be copied among the agents that produce the same good and have the highest score. In practice, the worst (in terms of score) 20 percent of the agents producing the same good will copy the prices of the best twenty percent producing the same good. This selection process is detailed in algorithm 2 .

Trading During the trading phase the value associated to a good by an agent corresponds to the subjective price of the good for this agent. Briefly summarised, for each good that it does not produce, an agent will trade with the first partner that offers an acceptable trade, i.e. an agent that proposes a satisfiable ratio between the other good and the good produced by the agent.

In more details, the trading phase starts by the agent looking at a first random agent producing another good. Let $o$ be an agent producing $g$ who proposes a trade and $r$ an agent producing $k$ that receives the proposition. As explained earlier, each has a quantity of good $Q^{o}$ and $Q^{r}$. On the one side, $o$ wants to exchange a quantity $w_{g}^{o}$ of the good $g$ for a quantity $w_{k}^{o}$ of the good $k$. On the other side, $r$ wants to exchange a quantity $w_{g}^{r}$ of the good $g$ for a quantity $w_{k}^{r}$. The tuples $W^{o}$ and $W^{r}$ describe the quantities of goods wanted by agent $o$ and $r$ for one trade proposition and are defined by: 


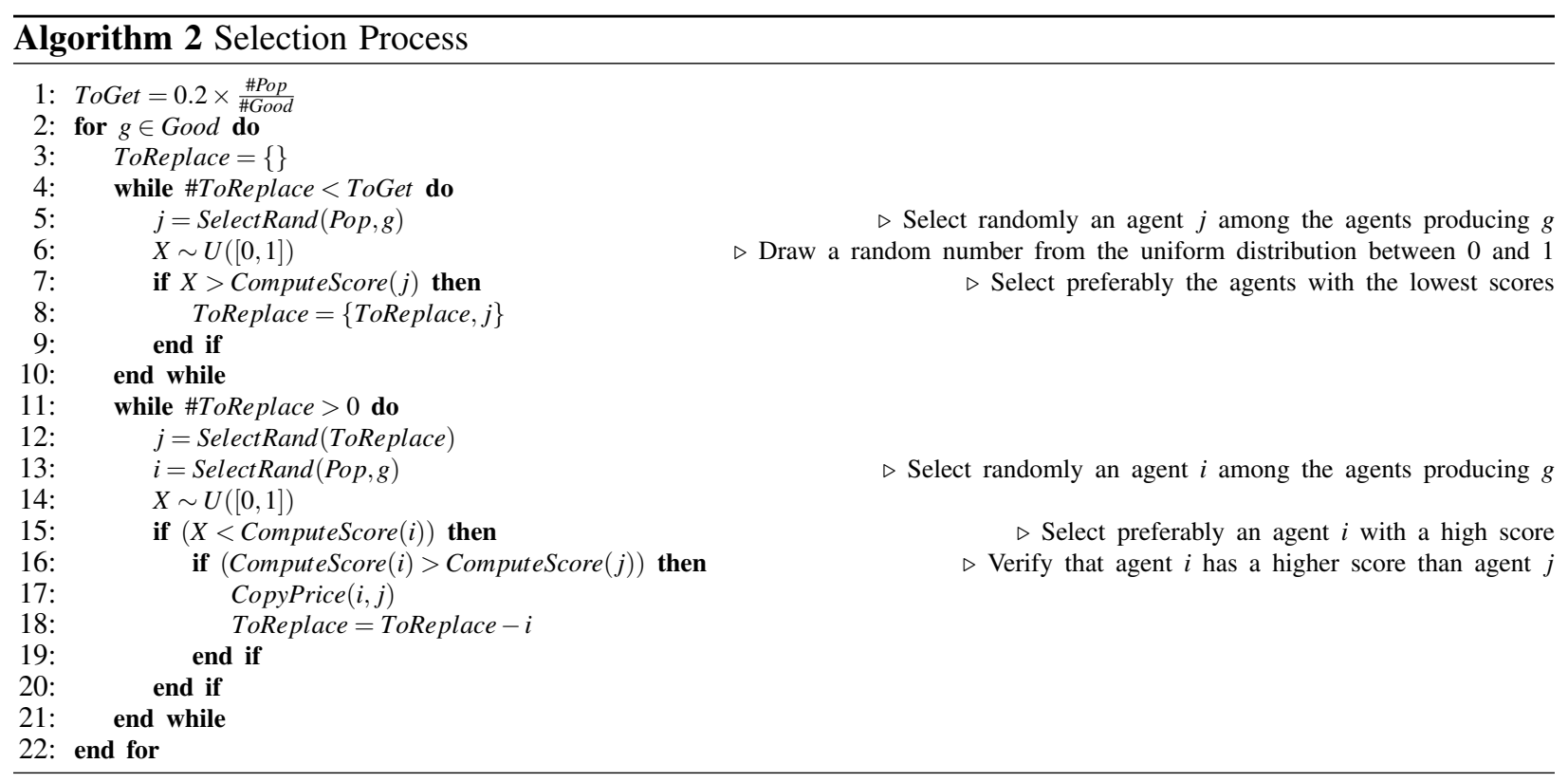

$$
W^{o}=\left(w_{g}^{o}=v_{g}^{o}, w_{k}^{o}=\frac{v_{k}^{o}}{v_{g}^{o}}\right), \quad W^{r}=\left(w_{k}^{r}=v_{k}^{r}, w_{g}^{r}=\frac{v_{g}^{r}}{v_{k}^{r}}\right)
$$

Where $v_{j}^{i}$ are the estimated value of the good $j$ by the agent $i$ as defined earlier. The requested quantity of the non produced good is simply the ratio between the estimated value of the good requested and the estimated value of the produced good.

Once the quantities are defined, the agents declare that the trade is possible if :

$$
\begin{array}{r}
q_{g}^{o}>=w_{g}^{o}, \quad q_{g}^{r}<=w_{g}^{o}, \quad q_{k}^{r}>=w_{k}^{o} \\
w_{g}^{o}>=\left(q_{g}^{r}+w_{g}^{r}\right), \quad w_{k}^{o}<=w_{k}^{r}, \quad w_{g}^{o}<=w_{g}^{r}
\end{array}
$$

The conditions 3 ensure that both agents have enough goods in their inventory to realise the trade while the conditions 4 ensure that the quantities of goods fit the will of both agents.

If a trade is possible, the two agents will exchange the agreed quantities. If the trade is not possible, the agent will continue to look at random partners for this good until either a partner is found or TradeThreshold agents have been tried. At this point the agent will try to trade with agents producing another good. The process goes on until all goods have been tried. This trading process is described in algorithm 3 .

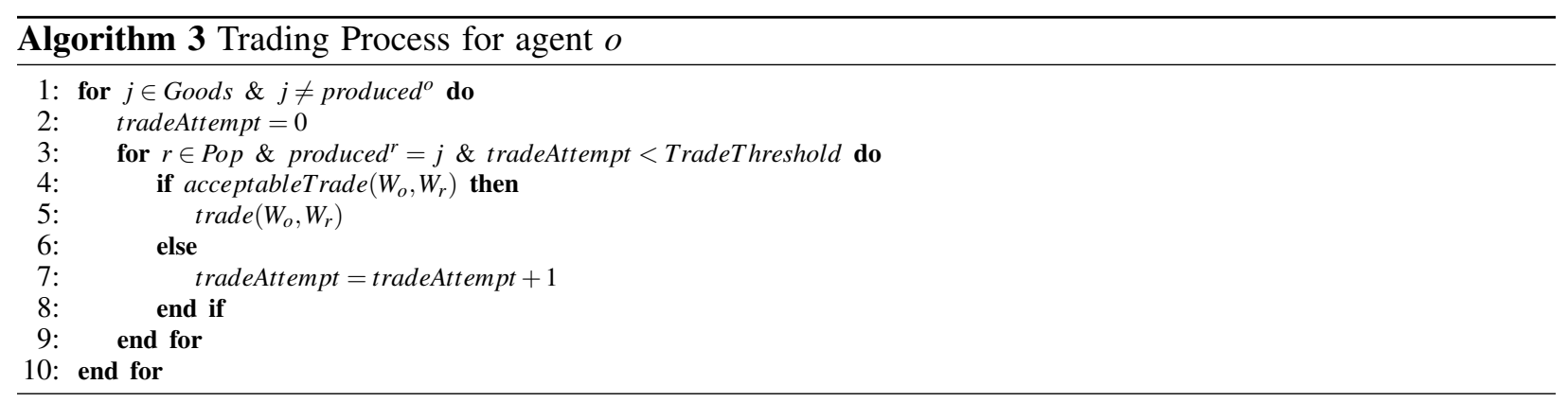

Innovation In a trading environment it seems unlikely that a price will change radically to a very different value. Therefore, a new and more realistic mechanism is proposed. The innovation process, coined "self referenced", is still triggered with probability $\mu$ but modifies the previous price by adding or subtracting a small amount taken randomly from a uniform distribution between $0 . . \beta$. 
Expected outcome Based on the set of implementations presented and given the equations (1) and (2), it is expected that the prices will converge to value allowing each agent to obtain quantities of resources exactly equivalent to the needs. The best possible price of all good satisfies the equations :

$$
\left\{\begin{array}{l}
\frac{v_{k}^{o}}{v_{g}^{o}}=n_{k} \\
v_{g}^{o}=n_{g}
\end{array} \quad=>v_{k}^{o}=n_{k} \times n_{g}, \quad \forall k \in \text { Goods }, \forall o \in \text { Pop }, g=\text { produced }^{o}, k \neq g\right.
$$

Which means that

$$
\forall j \in \text { Goods }, \forall i \in \text { Pop } \quad \tilde{V}^{i}= \begin{cases}\tilde{v}_{j}^{i}=n_{j} & \text { if } j=\text { produced }^{i} \\ \tilde{v}_{j}^{i}=n_{j} \times n_{\text {produced }^{i}} & \text { else }\end{cases}
$$

If such prices are reached, given the exchange rules defined in (2) and the exchange constraints (3) and (4) all exchanges will be optimally achieved, leading to a total score $S$ for each agent of the population :

$$
S=\sum_{i=0}^{\text {CulturalStep }} s^{i}\left(\tilde{Q}^{i}\right) \times \text { ngoods }
$$

where $\tilde{Q}^{i}$ is the optimal quantity vector, i.e. the one for which $s^{i}\left(\tilde{Q}^{i}\right)=s_{\max }$. Remember that from equation 1 , $s_{\max }=1$.

\subsection{Experimental setups}

The neutral model is tested through 15 experimental setups. The first six experimental setups are using 1 good, two population sizes (250 and 500 agents) and three values of $\mu(0.004,0.016$ and 0.064$)$. The remaining experimental setups are using 500 agents, 3 number of goods $(3,6$ and 9$)$ and three values of $\mu(0.004,0.016$ and 0.064$)$. For each setup, we have performed 100 runs of 10000 timestep each. The trading model is tested on an experimental setups using 3 goods, 500 agents and $\mu$ equal to 0.004 . Again 100 runs of 10000 timesteps are performed. The experiments, as well as the parameters used to run those experiments, are available online (Github Repository 2015) for the Pandora simulator (Rubio-Campillo 2014).

\section{RESULTS}

\subsection{Neutral Model}

We first analyse the result obtained in the "neutral model" with one good. The figure 1 presents the results obtained for two population sizes $N$ (250 and 500 agents) and $\mu$ varying through three values $(0.004,0.016,0.064)$. The figure is a log-log plot of the average (across all the runs) of the distribution of variants obtained through all experiments. The y-axis shows the proportions of the variants of the prices used during the simulation, the $\mathrm{x}$-axis shows how many variant achieves such proportions.

We observe that the lower the mutation rate, the closer to a line the result is. This line corresponds to the "power law" distribution explained in section 2.1, and is typical of the result obtained under the "neutral hypothesis". In order to verify if the distribution is in fact a power-law, we follow the method proposed by (Clauset, Shalizi, and Newman 2009) and the R implementation proposed by (Gillespie 2015). Briefly, two values are returned: a) the estimation of the $\alpha$ parameter of the power law equation $P(v)=C / v^{\alpha}$; ) a $p$-value testing the null-hypothesis that our data could have been generated from a power law distribution.

The table 1 summarizes the results obtained on all setups. Each value shown in the table is the mean values of 100 simulations. We see that in almost every case the null hypothesis cannot be rejected, which means that indeed the repartition of the price follows a power law. The only exception is for $\mu$ equal to 0.064 , where the $p$-value is less than 0.05 . In this last case the null hypothesis is rejected, and we therefore assume that the distribution does not follow a power law. 

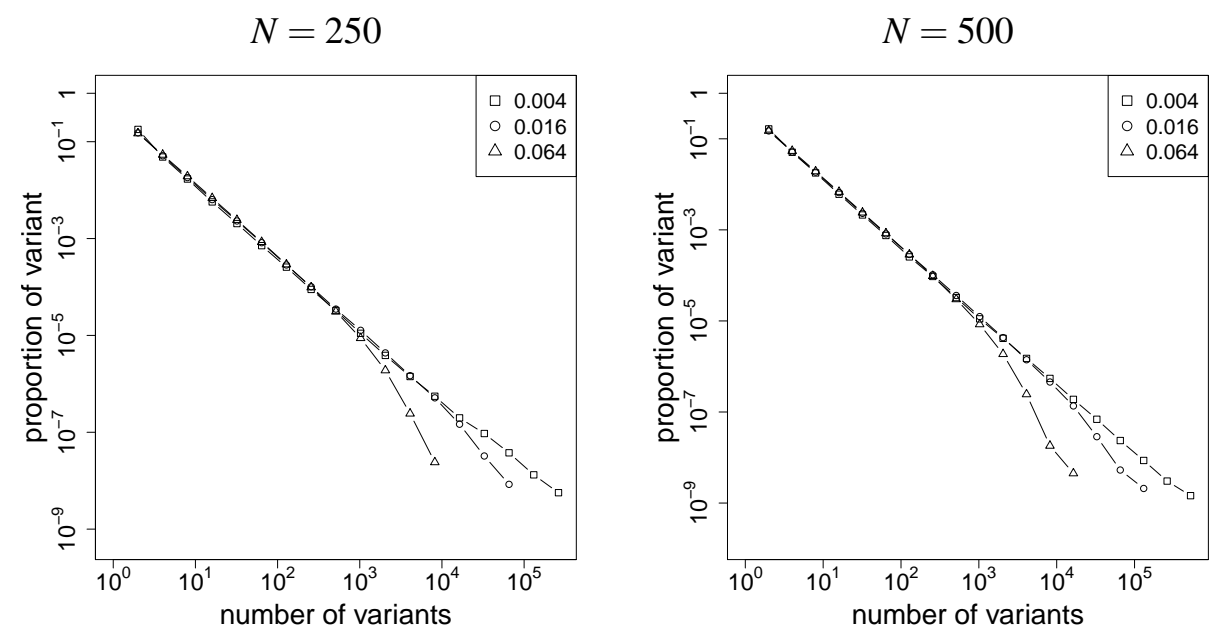

Figure 1: Distribution of proportions depending on the $\mu$ parameter with 250 agents (left) and 500 agents (right). Each plot is the mean obtenaid for 100 runs.

Table 1: Mean $\alpha \& s d$ are calculated on 100 runs for our results, and 5 runs for Bentley et. al. 2004. $p r$ is the percentage of run for which the $p$-value is less than 0.05 , i.e. the percentage of runs for which we rejected the null-hypotheses stating that the distribution follow a power law.

\begin{tabular}{cc|ccc} 
& & \multicolumn{2}{c}{ Our result } & Bentley et. al. 2004 \\
$\mathrm{N}$ & $\mu$ & $\alpha(s d)$ & $p$-value $(s d-p r)$ & $\alpha(s d)$ \\
\hline 250 & 0.004 & $1.53(0.03)$ & $0.58(0.24-.01)$ & $1.54(0.02)$ \\
& 0.016 & $1.57(0.02)$ & $0.35(0.28-.05)$ & $1.57(0.01)$ \\
& 0.064 & $1.66(0.01)$ & $0.0(0.00-1)$ & $1.67(0.01)$ \\
\hline 500 & 0.004 & $1.50(0.02)$ & $0.59(0.28-.02)$ & $1.53(0.03)$ \\
& 0.016 & $1.55(0.03)$ & $0.15(0.17-.10)$ & $1.61(0.04)$ \\
& 0.064 & $1.78(0.08)$ & $0.0(0.00-1)$ & $1.81(0.10)$
\end{tabular}

For comparison purposes the results obtained by (Bentley, Hahn, and Shennan 2004) (which tested the "neutral hypothesis" with the same methodology) are added in the last column of table 1. It appears that these results are highly similar to ours. We note also that our results match the ones presented in (Mesoudi and Lycett 2009) where only one value of $\mu$ was tested $(\mu=0.008)$. However, it is difficult to know if the slight differences observed between our work and those previous studies are statistically significant as the two previous studies rely on only five runs for the computation of the mean of $\alpha$ (against 100 in our case).

Nonetheless, for high values of $\mu$, previous works report that the distribution of variant follow a power law (Bentley, Hahn, and Shennan 2004). This claim is based on the fact that the estimated $\alpha$ (estimation based on linear regression on the log-log curve) has a high correlation coefficient. Recent works have shown that the use of correlation coefficient should be avoided (Clauset, Shalizi, and Newman 2009). Following these recent findings, our results preclude us to assume that the distribution of variant when $\mu$ is up to 0.064 follow a power law .

An additional series of experiments is done to analyse how the system reacts when multiple goods are present. The mean values of $\alpha$ and $p$-value have been analysed for 3 innovation rates $(0.004,0.016,0.064)$, 4 number of goods $(1,3,6,9)$, and 250 agents. However, due to the simplicity of the results and the space restriction we only report the analysis of the results here. Visual analysis reveals that independently of the number of goods, the distributions obtained are exactly the same. Therefore it is no surprise that, for each innovation rate, the properties of the distribution of prices does not depend on the number of goods. 


\subsection{Distribution of variants}

In order to understand the effect of introducing trading mechanisms, we compare first the distribution of values obtained in the "trading model" against the values obtained in the "neutral model". The figure 2.a) presents the results obtained from 100 runs for each model. All runs rely on the same experimental setup using 3 goods, 500 agents and $\mu$ equal to 0.004 . In all following graph a variant is one price of one given good. The distributions are first computed for each good independently and then averaged together.

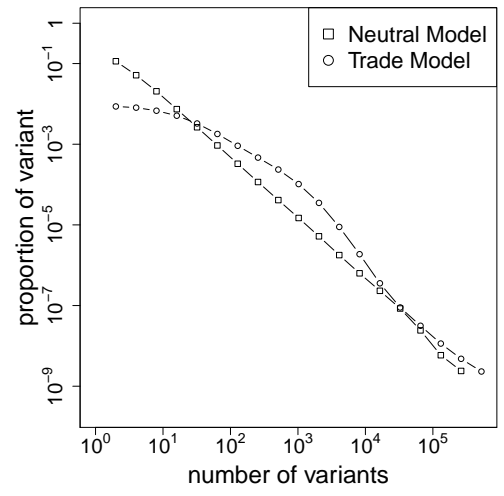

a)

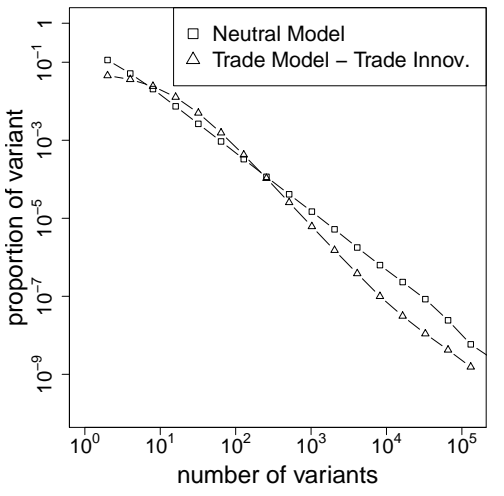

b)

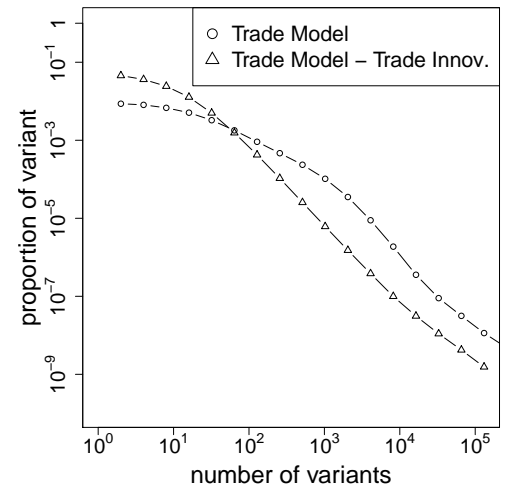

c)

Figure 2: Frequencies distribution, where each points represent the mean for 100 runs, for: a) the neutral and the trading models. b) the neutral model and the trading model without the trading innovation process. c) the trade model and the trade model without the trading innovation process.

On figure 2.a) it appears that the implementation of trade mechanisms leads to a distribution of prices departing from the neutral hypothesis. In more details, the frequencies distribution has a plateau of common prices (a number of prices share similar and high proportions), which shows that, when trade is taken into account, the most common variants are more diverse.

In order to investigate which mechanism influences this departure from the neutral model, additional investigations have been performed. Here is presented the results of the analysis on the effect of the innovation process of the trade model. To conduct this analysis the innovation process of the trade model has been replaced by the innovation process of the neutral model. 100 runs have been performed with this model on the same experimental setup. The results obtained are compared to the neutral model in figure 2.b) and to the trade model in figure 2.c).

On figure 2.b) it appears that the replacement of the innovation process leads to the creation of a distribution close to the one obtained with the neural model. On figure 2.c) we observe a strong reduction in the size of the plateau and an important difference between the two distributions. This analysis highlights the importance of the innovation process of the trading model in the distribution of prices. This mechanism, by preventing the creation of totally random new price, promotes the creation of few similar prices.

\subsection{Study of scores}

The results obtained with the trade model are studied in more detail by investigating the ability of the population to find the price most suited for exchanges. This is done by first measuring the score of all agents in each of the two different models. The figure 3 uses again the results obtained from 100 runs for each model where all runs rely on the same experimental setup using 3 goods, 500 agents and $\mu$ equal to 0.004. The figure is showing, as boxplots, the score computed thanks to equation (1) for all agents of all runs. The $y$-axis shows the score computed and the $\mathrm{x}$-axis shows the timesteps. The left plot shows the results obtained in the "neutral model" and the right plot shows the results obtained in the "trading model". 

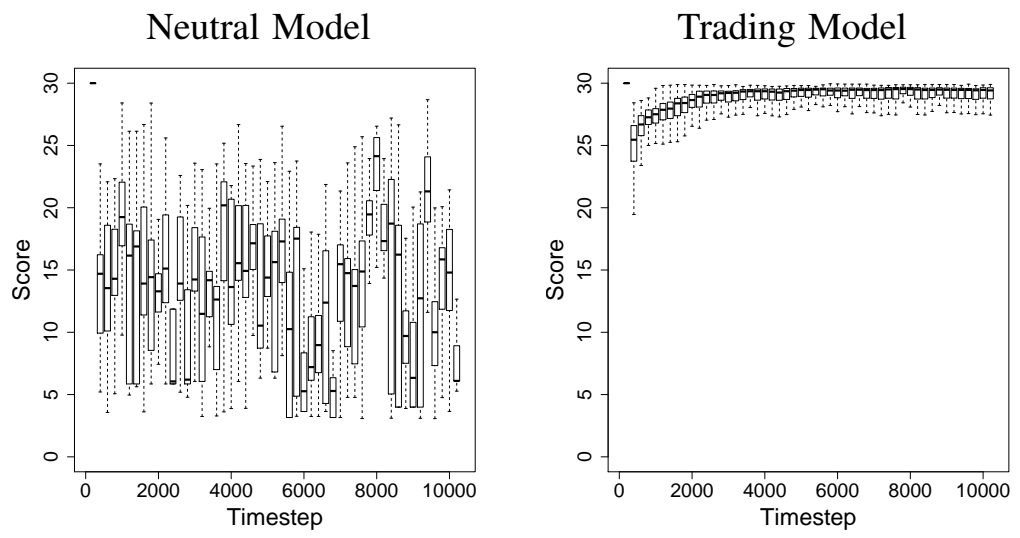

Figure 3: Evolution of the score within the two different models for two typical run with 500 agents and 3 goods evolving during 10000 timestep.

As expected, the scores within the neutral model vary randomly. "Trends" may appear, where a bigger proportion of individuals adopt a better price that allow agents to reach better score (such as around iteration 8000), but such good score fall back as soon as another trend appears. However, with the trading model, the score of all the agents increase. As the selection mechanism allow them to know who has found better vectors of prices, they will progressively adopt prices vector that allow all of them to reach better scores.

The previous figure showed the capacity for the trading model to increase its score but did not analyse the exact prices used. As explained in the section 2.2 we expect that the trade cultural transmission and innovation processes will produce a convergence toward a set of price for each good that will allow agents to exchange optimally the good they produce with the other goods. To verify this assumption we analyse the prices reached during the simulations. These are presented in figure 4 for the 100 runs relying the experimental setup using 3 goods, 500 agents and $\mu$ equal to 0.004 . For all runs, all agents and at each iteration we compute the difference between the price used by the agent $V_{g}$ and the optimal price $\tilde{V}_{g}$ (given by equation 6). The measures performed are presented as boxplots condensing the results for 100 runs.

We observe that prices are indeed converging to the optimal prices which means that the agents within the trading model are indeed improving their scores by reaching the optimal prices. Notably, a similar variation of prices was observed in the closely related economical model of (Gintis 2006). This variation of the prices to the optimum offers an additional conformation of the validity of the trading model.

\section{CONCLUDING REMARKS}

This article has proposed a framework to simultaneously study cultural change and trade dynamics. The development of our framework was first aimed at simplicity which is achieved by the use of two vectors (quantity and value) and five processes (production, consumption, trade, cultural transmission and innovation). The second aim of the work conducted was to obtain a flexible framework which is possible since each of the processes can be implemented accordingly to the question studied. We have shown the validity of this approach by reproducing expected results on both the cultural and trade side. On the cultural transmission side we have shown that the implementation of a "neutral model" leads to the expected observations on the variants of the vector value: a power law. When implementing trading mechanisms we observe the convergence of prices to the expected values and the improvement of the scores of the agents.

The successful implementation of a trading model within our framework shows that trading models can be viewed as a particular implementation of the cultural evolutionary framework. This aspect which has not been studied before, opens a new line of thinking on the weight of trade mechanisms within cultural change. Notably, we have shown that the implementation of trade mechanisms leads to a distribution of prices departing from the neutral hypothesis, which is the reference in the study of cultural changes. Within 


\section{Carrignon, Montanier, and Rubio-Campillo}

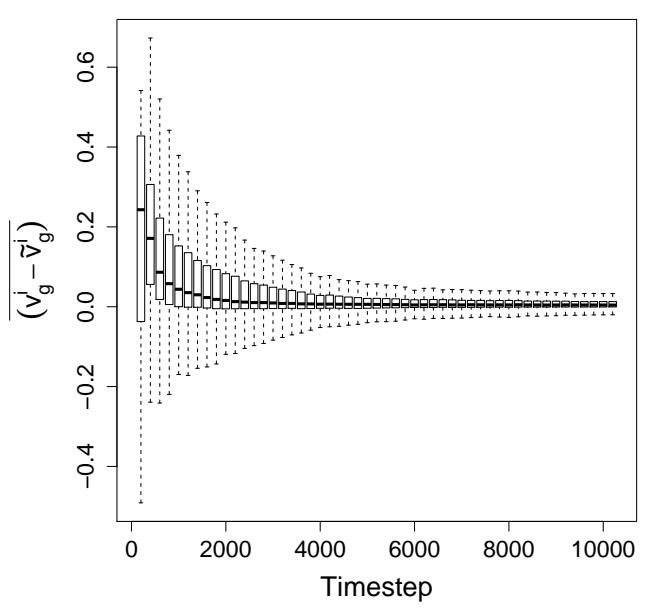

Figure 4: Evolution of the mean of the difference between the estimated value $v_{g}^{i}$ and the optimal value $\tilde{v}_{g}^{i}$ (calculated with equation 6) for a good $g$ and an agent $i$. As the optimal value $\tilde{v}_{g}^{i}$ depends on which good is produced by $i$, the mean of the difference between the estimated price and the optimal one is computed between all the agents that produce the same good. The figure represents this mean computed at each timestep for each goods, for each groups of agents and for 100 runs in a setup with 500 agents and 3 goods.

the trading model, the frequency distribution has a plateau of common prices (a number of prices share similar and high proportion), which shows that, when trade is taken into account, the most common variants are more diverse. Interestingly, we have not find references pointing at the ability of trade mechanisms to keep a relatively large diversity in the frequency distribution. We suspect that this is mainly because it is not common to compare results of trading models to other cultural evolution models. It would therefore be interesting to compare the frequency distributions obtained by our model and the ones observed in current or past economies. On the side of cultural transmission, the results obtained can also be compared to the ones obtained when prestige biased cultural transmission is used. The idea behind this last comparison, is that trade could be interpreted as a particular prestige biased cultural transmission and therefore be fully integrated within the cultural evolutionary frameworks.

In future works more realistic scenarios can be studied in the same framework. Multiple implementations of the production mechanism can be studied so as to increase the number of goods per agent and include factors such as the meteorology or the various type of goods. Moreover, the introduction of the distinction between "vital" and "common" goods will be useful for the creation of models studying in further details the interaction between trade and cultural transmission.

Additionally, more complex dynamics can be looked for. The trading mechanism can be naturally implemented in different ways, each reflecting a specific theory, and thus allowing their comparisons. The cultural transmission mechanism also can be modified by including for example a crossover between the cultural trait of multiple agents. The trade network (which in this work can be interpreted as fully connected) can also be modified to study the effect of slow connections or the rupture of certain connections. The agent themselves could become more complex and be endowed with the ability to learn behaviours which would again produce more realistic simulations. Moreover, various populations can be envisioned, each with their distinctive characteristics regarding cultural transmission and trade.

Finally, in terms of analysis of the results, multiple factor could be taken into account. The rapidity of the fluctuation of prices is a good starting point for the establishment of economical studies. For the study of a population of agent in general, it can be interesting to analyse the number of agents active and the composition of the population. 
Carrignon, Montanier, and Rubio-Campillo

\section{ACKNOWLEDGEMENTS}

Funding for this work was provided by the ERC Advanced Grant EPNet (340828) and the SimulPast Consolider Ingenio project (CSD2010-00034) of the former Ministry for Science and Innovation of the Spanish Government.

The model was created using Pandora (Rubio-Campillo 2014). R was used for figures and statistical analysis (R Development Team 2012). The estimation of the $\alpha$ parameters have been computed with the $\mathrm{R}$ package poweRlaw (Gillespie 2015). The simulations have been done in the supercomputer MareNostrum at Barcelona Supercomputing Center - Centro Nacional de Supercomputación (The Spanish National Supercomputing Center). The source code of the model is licensed under a GNU General Public License.

\section{REFERENCES}

Bentley, R. A., M. W. Hahn, and S. J. Shennan. 2004. "Random drift and culture change". Proceedings of the Royal Society of London. Series B: Biological Sciences 271 (1547): 1443-1450.

Bentley, R. A., M. W. Lake, and S. J. Shennan. 2005. "Specialisation and wealth inequality in a model of a clustered economic network". Journal of Archaeological Science 32 (9): 1346-1356.

Boyd, R., and P. J. Richerson. 2005. The origin and evolution of cultures. Oxford University Press.

Brughmans, T. 2010. "Connecting the dots: towards archaeological network analysis". Oxford Journal of Archaeology 29 (3): 277-303.

Clauset, A., C. R. Shalizi, and M. E. Newman. 2009. "Power-law distributions in empirical data". SIAM review 51 (4): 661-703.

Crema, E., K. Edinborough, T. Kerig, and S. Shennan. 2014. "An Approximate Bayesian Computation approach for inferring patterns of cultural evolutionary change". Journal of Archaeological Science 50:160170.

Gillespie, C. S. 2015. "Fitting Heavy Tailed Distributions: The poweRlaw Package". Journal of Statistical Software 64 (2): 1-16.

Gintis, H. 2006. "The emergence of a price system from decentralized bilateral exchange". Contributions in Theoretical Economics 6 (1): 1-15.

Github Repository 2015. "Github Repository”. Accessed July 22, 2015. https://github.com/montanier/ CMR-WSC-CoEvolutionTradeCulture.

Henrich, J., and R. McElreath. 2003. "The evolution of cultural evolution". Evolutionary Anthropology: Issues, News, and Reviews 12 (3): 123-135.

Heyes, C. M. 1994. "Social learning in animals: categories and mechanisms". Biological Reviews 69 (2): 207-231.

Kandler, A., and S. Shennan. 2013. "A non-equilibrium neutral model for analysing cultural change". Journal of Theoretical Biology 330:18-25.

Lake, M. W. 2014, June. "Trends in Archaeological Simulation”. Journal of Archaeological Method and Theory 21 (2): 258-287.

Lipo, C. P., and M. Madsen. 2001. "Neutrality,"style", and drift: building methods for studying cultural transmission in the archaeological record". In Style and Function: Conceptual Issues in Evolutionary Archaeology, edited by T. D. Hurt and R. Gordon F.M., 91-118. Bergin and Garvey.

Macmillan, W., and H. Huang. 2008, March. "An agent-based simulation model of a primitive agricultural society". Geoforum 39 (2): 643-658.

Mesoudi, A., and S. J. Lycett. 2009, January. "Random copying, frequency-dependent copying and culture change". Evolution and Human Behavior 30 (1): 41-48.

Neiman, F. D. 1995. "Stylistic Variation in Evolutionary Perspective: Inferences from Decorative Diversity and Interassemblage Distance in Illinois Woodland Ceramic Assemblages". American Antiquity 60 (1): pp. 7-36. 
Porčić, M. 2014. "Exploring the Effects of Assemblage Accumulation on Diversity and Innovation Rate Estimates in Neutral, Conformist, and Anti-Conformist Models of Cultural Transmission". Journal of Archaeological Method and Theory:1-22.

R Development Team 2012. "R: A language and environment for statistical computing".

Remesal, J., A. Díaz-Guilera, B. Rondelli, X. Rubio-Campillo, A. Aguilera, D. Martín-Arroyo, A. Mosca, and G. Rull. 2014. "The EPNet Project. Production and distribution of food during the Roman Empire: Economics and Political Dynamics". In Information Technologies for Epigraphy and Cultural Heritage: Proceedings of the First EAGLE International Conference, 455-464. Paris, France: Sapienza Universitá.

Rubio-Campillo, X. 2014. "Pandora: A Versatile Agent-Based Modelling Platform for Social Simulation". In Proceedings of SIMUL 2014, The Sixth International Conference on Advances in System Simulation, 29-34. IARIA Publishing.

Schillinger, K., A. Mesoudi, and S. Lycett. 2014. "Copying error and the cultural evolution of "additive" vs."reductive" material traditions: an experimental assessment". American Antiquity 79 (1): 128-143.

Shennan, S. J., and J. R. Wilkinson. 2001. "Ceramic Style Change and Neutral Evolution: A Case Study from Neolithic Europe". American Antiquity 66 (4): 577.

Solé, R., S. Valverde, M. R. Casals, S. A. Kauffman, D. Farmer, and N. Eldredge. 2013. "The evolutionary ecology of technological innovations". Complexity 18 (4): 15-27.

Steele, J., C. Glatz, and A. Kandler. 2010. "Ceramic diversity, random copying, and tests for selectivity in ceramic production". Journal of Archaeological Science 37 (6): 1348 - 1358.

Temin, P. 2001. "A market economy in the early Roman Empire". Journal of Roman studies 91:169-181. Ziman, J. 2003. Technological innovation as an evolutionary process. Cambridge University Press.

\section{AUTHOR BIOGRAPHIES}

SIMON CARRIGNON is a PhD student in Computer Applications in Science and Engineering, Barcelona Supercomputing Center. He holds a M.S. in Cognitive Sciences and a M.A. in History and Philosophy of Science. His works focused on the use of computer modeling and evolutionary method to study Complex Systems, ranging from Evolutionary Biology to Human and Cultural Dynamics. His email address is simon.carrignon@bsc.es.

JEAN-MARC MONTANIER is Researcher in Computer Applications in Science and Engineering, Barcelona Supercomputing Center. He has obtained his engineering title in the domain of embedded computing and holds a Ph.D. in collective robotics. His topics of interest focused on Agent-Based Modeling, swarm adaptation, learning of cooperative strategies, division of labour and more recently study of economical and cultural evolution. His email address is jean.montanier@bsc.es.

XAVIER RUBIO-CAMPILLO is Researcher in Computer Applications in Science and Engineering, Barcelona Supercomputing Center. He has a transdisciplinary training of Computer Science (BSc) and Humanities $(\mathrm{PhD})$ with a strong emphasis on the study of past societies using historical and archaeological contexts. His main research line is the application of formal models to explore the processes of cultural evolution in past societies. His email address is xavier.rubio@bsc.es. 